\title{
Die Zulässigkeit der Übertragung von Managementaufgaben auf Chefärzte
}

\section{Einführung \\ $\nabla$}

Der Tätigkeitsbereich des Chefarzts im Krankenhaus wird bereits seit einiger Zeit nicht mehr nur aus seiner medizinischen Funktion abgeleitet, sondern er umfasst in immer weiter zunehmendem Maße auch Managementaufgaben. Dies kann in vielen Bereichen gar nicht beanstandet werden, da sich die Leitung einer Abteilung, eines Instituts oder einer Klinik sicherlich auch für vielfältige Aufgabenzuweisungen eignet, die den administrativen Bereich bzw. die Managementebene betreffen. Letztlich werden in letzter Zeit jedoch vermehrt Aufgaben auf Chefärzte verlagert, die entweder einer direkten Zuweisung an den Krankenhausträger als Arbeitgeber unterliegen oder die aufgrund der systemeigenen Abläufe gar nicht sinnvoll zuweisbar sind. Des Weiteren werden in den Dienstverträgen verschiedene Formulierungen verwendet, die zu gewünschten nebendienstlichen Tätigkeiten eigentlich im Widerspruch stehen.

\section{Strahlenschutzverantwortlicher und / oder Strahlenschutzbeauftragter $\nabla$}

Einige Häuser kommen auf die Idee, den radiologischen Chefarzt zum Strahlenschutzverantwortlichen zu „ernennen“. Festzuhalten ist, dass dies aus gesetzlicher Sicht nicht möglich ist. Gem. § 13 RöV ist derjenige der Strahlenschutzverantwortliche, der eine Genehmigung nach $\S 3$ oder $\S 5$ RöV benötigt bzw. derjenige, der eine Anzeige nach $\S 4$ RöV zu erstatten hat. Zwar wird über $\S 6$ Abs. 2 RöV klargestellt, dass auch Hersteller und Wartungsunternehmen, die Tätigkeiten im Sinne von $\S 6$ Abs. 1 Nr. 1 und Nr. 2 RöV ausüben, unter $\S 13$ RöV fallen und auch Ärzte, die an Fremdanlagen eine Tätigkeit nach $\S 6$ Abs. 1 Nr. 3 RöV ausüben, umfasst sind, jedoch bleibt es generell dabei, dass Strahlenschutzverantwortlicher der Betreiber der Anlage ist. Insoweit handelt es sich um den Besitzer der Anlage. Bei einem Krankenhaus ist dies der Krankenhausträger, welcher je nach gewählter Rechtsform zumeist durch die zur Vertretung berechtigten Personen abgebildet wird. Insoweit durch den Vorstand bzw. die Geschäftsführer.

Der oben dargestellte Versuch aus der Praxis, die Stellung des Strahlenschutzverantwortlichen zu delegieren, kann insoweit nicht gelingen, selbst wenn zuzugestehen ist, dass der Strahlenschutzverantwortliche die Wahrnehmung seiner Aufgabe im Rahmen der Betriebs- und Verwaltungsorganisation an einen Vertreter delegieren kann. Dieser muss nicht die Stellung des Strahlenschutzbeauftragten haben. Eine diesbezügliche Delegation führt jedoch ebenfalls nicht zur „Ernennung“ des Vertreters zum Strahlenschutzverantwortlichen. Der Krankenhausträger ist trotz der Bestimmung eines Vertreters weiterhin der Betreiber der Anlage und somit nach $\S 13$ RöV Strahlenschutzverantwortlicher.

Zweifelsfrei ist es jedoch möglich, dass der Strahlenschutzverantwortliche den radiologischen Chefarzt zum Strahlenschutzbeauftragten ernennt (vgl. § 13 RöV und $\S 31$ StrlSchV). Der Strahlenschutzbeauftragte ist eine fachlich kundige Person, die im Betrieb dafür verantwortlich ist, dass ein Gerät an seinem Aufstellungsort auch tatsächlich sicher betrieben wird (Schmatz/Nöthlichs, Strahlenschutz Lfd. 8224 S. 5). Zu berücksichtigen hierbei ist, dass der nicht fachkundige Strahlenschutzverantwortliche - dies dürfte in der Regel der Fall sein - gemäß $\S 13$ Abs. 2 S. 1 RöV bzw. $§ 31$ Abs. 2 S. 1 StrlSchV zwingend eines Strahlenschutzbeauftragten für die Sicherung des Betriebs bedarf. In diesem Fall muss der radiologische Chefarzt in seiner Funktion als Strahlenschutzbeauftragter wissen, dass er bei notwendigen Aufgaben des Strahlenschutzes keinen Weisungen des Strahlenschutzverantwortlichen unterliegen kann. Hintergrund ist, dass der Strahlenschutzbeauftragte, mit Wahrnehmung seiner Aufgaben nach beispielsweise $\S 15$ Abs. $1 \mathrm{Nr} .3$ oder $§ 15$ Abs. 2 RöV eine öffentlich-rechtliche Pflicht wahrnimmt (Schmatz / Nöthlichs, Strahlenschutz Lfd. 8224 S. 5), wobei eine grundsätzliche Organisationshoheit des Strahlenschutzverantwortlichen jedoch verbleibt.
Der radiologische Chefarzt dürfte in der Regel zu einem der Strahlenschutzbeauftragten bestellt werden. $\mathrm{Zu}$ beachten ist jedoch, dass dies schriftlich zu erfolgen hat und die Aufgaben, der innerbetriebliche Entscheidungsbereich und die hiermit verbundenen Befugnisse festzulegen sind. Dies erfolgt im Interesse beider Parteien. Neben den gesetzlichen Vorgaben ist dies auch vor dem Hintergrund notwendig, dass der radiologische Chefarzt nicht für die Einhaltung der Vorschriften in den von ihm nicht chefärztlich kontrollierten Bereichen zuständig sein sollte.

\section{Verantwortlicher für Maßnahmen nach dem Arbeitsschutzgesetz $\nabla$}

In aktuellen Chefarztverträgen wird zudem immer häufiger die Verantwortlichkeit nach dem Arbeitsschutzgesetz auf den Chefarzt delegiert.

Festzuhalten ist zunächst auch hier, dass der Adressat der gesetzlichen Regelung gemäß § 3 Abs. 1 ArbSchG nicht der Chefarzt, sondern der Arbeitgeber und somit der Krankenhausträger ist. $\S 13$ ArbSchG enthält eine abschließende Aufzählung der verantwortlichen Personen. Der Chefarzt ist hier nicht genannt und kann zudem nicht unter die mit der Leitung eins Unternehmens oder Betriebes beauftragten Personen nach $\S 13$ Abs. 1 Nr. 4 ArbSchG fallen, da hierzu die dem Chefarzt zugebilligten personalrechtlichen Entscheidungskompetenzen in der Regel nicht ausreichen dürften.

Dennoch könnte ein Chefarzt arbeitsvertraglich zur Aufsicht über die Einhaltung des Arbeitsschutzgesetzes beauftragt werden, sodass eine Delegation der auszuführenden Überwachungen und Maßnahmen möglich erscheint, sofern dies nicht im Widerspruch zu seinen chefärztlichen Aufgaben steht. Voraussetzung ist jedoch, dass der individuelle Chefarzt zwingend als „fachkundige Person“ gemäß 113 Abs. 2 ArbSchG anzusehen wäre. 
Nur dann wäre die Tätigkeit „in eigener Verantwortung“ des Chefarzts möglich. Dies dürfte bei einem Chefarzt ohne ausdrückliche Schulung im Arbeitsschutz fraglich sein. Denn die Aufgabe des Chefarzts wäre unter anderem die Durchführung der Gefährdungsbeurteilung nach dem Arbeitsschutzgesetz und die Erstellung von Arbeitsanweisungen als Standard. Nicht zuletzt sind zudem oft die Anschaffung und Unterhaltung der Funktionsfähigkeit der Arbeitsschutzmittel vertraglich umfasst, welche der Chefarzt jedoch in der Regel nicht ohne Freigabe der Geschäftsführung anschaffen kann, was die Verantwortlichkeit konterkariert.

Allerdings ist es möglich, den Chefarzt auch als Nichtverantwortlichen in die gesetzlichen Aufgaben nach dem ArbSchG einzubinden. Jedoch entbindet dies die nach dem Gesetz verantwortlichen Personen nicht, welche auch weiterhin der sie persönlich treffenden Aufsichts- und Organisationspflicht nachkommen müssen. Ob sich diese Pflicht im Falle einer zulässigen Delegation auf stichprobenartige Überprüfungen des Beauftragten reduzieren lässt, ist zunächst fraglich, jedoch im Einzelfall zu hinterfragen.

Letztlich muss festgestellt werden, dass der Arbeitgeber mit der Delegation eine reine Arbeitgeberverpflichtung auf einen seiner eigenen Arbeitnehmer stellungsfern überträgt. Die Wahrnehmung direkter Arbeitgeberpflichten durch eigene und möglichst selbständig tätige Arbeitnehmer unterliegt dabei umfassenden Kontrollpflichten durch den Arbeitgeber, sodass unzureichende Maßnahmen überarbeitet werden müssen. Da der Chefarzt nach $\S 15$ ArbSchG bereits auf den ihn selbst betreffenden Arbeitsschutz und nach $\S 15$ Abs. 1 S. 2 ArbSchG auch auf die Personen zu achten hat, die von seinen Handlungen oder Unterlassungen betroffen sind, nach $\S 16$ Abs. 1 ArbSchG einer unverzüglichen Unterrichtungspflicht über festgestellte unmittelbare erhebliche Gefahren für die Sicherheit und Gesundheit und jeden an den Schutzsystemen festgestellten Defekt unterliegt und schließlich nach $\S 16$ Abs. 2 S. 1 ArbSchG bereits eine Unterstützungspflicht für die Sicherheit und den Gesundheitsschutz der Beschäftigten bei der Arbeit besteht, dürften weitere arbeitsvertragliche Regelungen für einen fachunkundigen und somit nichtverantwortlichen Chefarzt größtenteils deklaratorischen Charakter besitzen.

\section{Medizinprodukteverantwortlicher} 7

Eine in dem Anstellungsvertrag oft nicht oder nur beiläufig benannte und später mittels Dienstanweisung zugewiesene Position ist die des Medizinprodukteverantwortlichen nach $\S 5$ Abs. 2 MPBetreibV. Medizinprodukteverantwortliche sind in ihrem Zuständigkeitsbereich verantwortlich für die Umsetzung und Einhaltung des Medizinproduktegesetzes (MPG), der Medizinprodukte-Betreiberverordnung (MPBetreibV) und der MedizinprodukteSicherheitsplanverordnung (MPSV).

Der betroffene radiologische Chefarzt wird hierdurch jedoch nicht nur Verantwortlicher für die MRT, CT und Röntgenanlage seiner Abteilung, sondern auch für jedes weitere Medizinprodukt, welches in der Abteilung - unter Umständen auch nur einmalig - verwendet wird. Der Medizinprodukteverantwortliche ist daher innerhalb seines Zuständigkeitsbereichs verpflichtet, für die Umsetzung der Einhaltung des Medizinproduktegesetzes mit seinen Rechtsverordnungen und unter Einbezug der entsprechenden Richtlinien zu sorgen. Dabei ist zu beachten, dass der Zuständigkeitsbereich nicht automatisch auf die Abteilung des Chefarzts begrenzt sein muss. Durchaus denkbar wäre es, dass der radiologische Chefarzt als Medizinprodukteverantwortlicher auch auf den beispielsweise im OP eingesetzten mobilen C-Bogen zu achten hat.

Sofern also der Chefarzt tatsächlich zum Medizinprodukteverantwortlichen ernannt werden soll, sind die Pflichten und Grenzen seiner Tätigkeit aus für beide Seiten geltenden Sicherheitsaspekten schriftlich in eindeutiger Form niederzulegen.

Aus rechtlichen Gesichtspunkten muss dem betroffenen Chefarzt klar sein, dass er eine Vielzahl der dem Krankenhausträger als Betreiber des Medizinprodukts obliegenden Pflichten verantwortlich wahrzunehmen hat. Oft wird seitens des Chefarzts verkannt, dass mit der Übernahme der Position und der hiermit verbundenen Aufgabenübertragung auch haftungsund strafrechtliche Risiken beinhaltet sind.

Festzuhalten ist, dass die Position des Medizinprodukteverantwortlichen durch einen Chefarzt ohne eine umfangreiche Schulung kaum möglich sein wird. Der durchschnittliche Chefarzt dürfte in der Regel die Vorgaben aus dem Medizinpro- duktegesetz und der sich hieraus ableitenden Medizinprodukteverordnungen bzw. die rechtlichen Definitionen beispielsweise von Inverkehrbringen, Prüfen, Erstellen und Bearbeiten nicht abschließend kennen. Allein dies verdeutlicht, dass vor der Übernahme der Position aus allseitigen Sicherheitsaspekten ein umfassender Erkenntnisgewinn einzutreten hätte.

\section{Zurverfügungstellung der gesamten Arbeitskraft \\ $\nabla$}

Auf den ersten Blick stellt die Formulierung: „Der Chefarzt stellt seine gesamte Arbeitskraft für seine Dienstaufgaben zur Verfügung“ keinen direkten Bezug zu zusätzlichen Tätigkeiten des Chefarzts dar. Jedoch ist zu berücksichtigen, dass das etwaig eingeräumte Liquidationsrecht eine zusätzliche Tätigkeit ist, deren zugrunde liegende Leistungserbringung keine Dienstaufgabe darstellt. Insoweit bestünde ein Konflikt innerhalb der Pflichten und Befugnisse des Chefarzts, der aus Rechtssicherheitsaspekten zwingend umgangen werden sollte.

Bei einer Verwendung dieser Klausel könnte es im Streitfalle zu einer Auseinandersetzung über die Umfänge der für die Liquidationsmöglichkeit notwendigen Leistungserbringung kommen, welche mit Entfall der Klausel deutlich reduziert werden.

Im engen Zusammenhang zu einem eingeräumten Liquidationsrecht steht auch die in vielen Verträgen enthaltene Freistellungsklausel bei etwaigen Kündigungen. Derartige Klauseln sind bei ordnungsgemäßer Formulierung in der Regel zulässig. Zwar kann der Chefarzt versuchen über ein, den vertraglichen Freistellungsanspruch übersteigendes, Interesse an der Weiterbeschäftigung aufgrund von erheblichen Beeinträchtigungen seines ärztlichen Fortkommens oder der aufrechtzuerhaltenden ärztlichen Fähigkeiten, dieses im Anwendungsfall zu umgehen, jedoch obliegen dem Arzt sodann die strengen Beweispflichten.

Im Zusammenhang mit der Freistellungklausel ist jedoch zwingend zu berücksichtigen, dass der Arbeitgeber diejenige Vergütung zu zahlen hat, die der betroffene Arzt während einer angenommenen weiteren Tätigkeit hätte erzielen können. Das Bundesarbeitsgericht (BAG Urt. v. 
18.09.2002 Az.: 1 AZR 686/01) hat bereits entschieden, dass hierbei sämtliche Entgeltbestandteile $\mathrm{zu}$ berücksichtigen sind. Vergütungsmindernde Freistellungsklauseln sind daher zwingend auf ihren Inhalt zu überprüfen.

\section{Verantwortlicher für die DRG-Kodierung}

In den meisten ärztlichen Anstellungsverträgen ist geregelt, dass der Arzt für die richtige DRG-Kodierung und deren Dokumentation verantwortlich ist. Sofern dies im Vertrag geregelt ist, besteht kaum eine rechtliche Möglichkeit, die Dienstaufgabe nachträglich anzugreifen. In einer arbeitsgerichtlichen Auseinandersetzung würde die chefärztliche Verantwortung für die vollständige Kodierung und Dokumentation abschließend feststehen.

Das Landesarbeitsgericht Sachsen (LAG Sachsen Urt. v. 01.12.2010 Az.: 2 Sa 56/10) hat bereits eine Kündigung - nach erfolgter Abmahnung - eines Chefarzts wegen unterlassener Kodierung für zulässig und sozial gerechtfertigt erachtet.

Die im Vertrag befindliche Klausel zur Aufnahme und Dokumentation der Kodierung sollte somit beide Vertragsparteien für sich versuchen optimal zu gestalten. Des Weiteren sollte ein betroffener Chefarzt bei der Verhandlung über die notwendige Umsetzung nachdenken und etwaige personelle Konsequenzen hieraus im ebenfalls zu verhandelnden Stellenplan berücksichtigen. In kodierungsaufwändigen Fachgebieten kann sich hier ein notwendiger Verhandlungsspielraum ergeben.

\section{Ergebnis \\ $\nabla$}

Die ohnehin vielfältigen Tätigkeitsbereiche eines Chefarzts und auch diejenigen der Oberärzte bedürfen zweifelsfrei einer rechtssicheren Basis, welche der Dienstvertrag und die dienstrechtlichen Weisungen zu bilden haben. Treten zu diesen Tätigkeiten weitere Managementaufga- ben hinzu, sind diese zwingend auf eine zulässige bzw. sinnvolle Delegation zu überprüfen und müssen rechtssicher in den Dienstvertrag bzw. das Dienstverhältnis implementiert werden. Beide Seiten sollten bezüglich der abschließenden Zuweisung auf eine umfassende Schriftlichkeit bestehen. Nur wenn die Pflichten, aber auch die Grenzen der Tätigkeit klar sind, können sowohl der Arzt als auch der Krankenhausträger sicher sein, dass die Inhalte der Aufgabe abschließend beidseitig verstanden wurden und somit erfüllbar sind.

Jens Remmert, LL.M. Rechtsanwalt

\section{Rechtsanwälte Wigge}

Scharnhorststr. 40

48151 Münster

Telefon: (0251) 53595-0

Telefax: (0251) 53595-99

E-Mail: kanzlei@ra-wigge.de

www.ra-wigge.de 